Submission ID: 32403

\title{
Robust Non-Stationary Signature Deconvolution
}

\section{M.S. Denisov* (GEOLAB), A.E. Firsov (GEOLAB)}

\section{SUMMARY}

Signature deconvolution is a widely used tool in seismic data processing. Sometimes its application leads to severe noise increase. This disadvantage is explained by the fact that filter adaptation is performed to the theoretical or measured signature, as the filter is subsequently applied to the data recorded. To achieve robust filtering, a data-adaptive element should be incorporated into the scheme. A threshold-based criterion is introduced to detect noisy frequencies and a special compensation filter is designed to attenuate them. The efficiency of the algorithm is demonstrated on a real marine data example 


\title{
Устойчивая нестационарная адаптивная сигнатурная деконволюция
}

\author{
Денисов М.С.* (ООО «ГЕОЛАБ»), Фирсов А.Е. (ООО «ГЕОЛАБ»)
}

\section{Введение}

При обработке материалов сейсморазведки применяют так называемую деконволюцию по форме импульса (Боганик, Гурвич, 2006). Часто эту процедуру также называют сигнатурной деконволюиией, и такое уже устоявшееся наименование является транслитерацией английского выражения signature deconvolution. В отличие от статистических алгоритмов, когда оценка импульса извлекается непосредственно из сейсмических трасс, такую деконволюцию можно считать детерминистической. В самом деле, подразумевается, что сейсмическая трасса описывается одномерной сверточной моделью, и доступна оценка формы импульса, которая может быть рассчитана теоретически при помощи соответствующих программ или измерена в процессе сбора данных. Применение процедуры не ограничивается лишь случаем морских наблюдений, и сигнатурная деконволюция используется также и в наземной сейсморазведке. В частности, известны методики обработки данных вибросейсмических наблюдений, когда вместо корреляции с опорным свип-сигналом производится деконволюция (Шехтман, Кузнецов, 2005), или когда форма импульса извлекается в процессе стратиграфической деконволюции, и затем обратный фильтр применяется к данным (Гогоненков, 1987).

При обработке сейсмограмм, полученных в результате морской сейсморазведки, сигнатура включает в себя, помимо прочих факторов, волны-спутники со стороны источника и приемника. Наличие спутников, устранение которых является одной из основных целей сигнатурной деконволюции, затрудняет ее использование. Анализируя эту ситуацию, Л. Хаттон и его соавторы (Хаттон и др., 1989) приходят к следующему выводу: «Волны-спутники .... - это камень преткновения детерминистической деконволюции. На первый взгляд кажется, что для подавления волн-спутников достаточно просто рассчитать обратный фильтр и применить его к данным. На самом деле это не так по нескольким причинам.

1. Амплитудный спектр имеет глубокий провал на частоте, определяемой глубиной источника. Полная компенсация такого провала потребует резкого усиления компонент в узком частотном диапазоне.

2. Вариации глубины источника в ходе разведки приводят к смещениям провала в спектре.

3. Возможны также значительные вариации коэффициента отражения водной поверхности, которые зависят от волнении моря и других факторов.....

Приведенные соображения способны охладить пыл самого ревностного приверженца детерминистической деконволюции».

На наш взгляд, описанная проблема обусловлена тем, что настройка фильтра сигнатурной деконволюции происходит по модельному или измеренному импульсу, а применение этого фильтра производится к полевым сейсмограммам. В последних могут содержаться помехи различной природы, которые невозможно учесть при получении импульса. Фильтр рассчитывается при помощи средств решения обратных задач, тем самым его применение может приводить к неустойчивости. В частности, в приведенной выше цитате указывается на наличие глубоких провалов в спектре исходного импульса, обусловленных волнамиспутниками. Обратный фильтр, стремясь выровнять амплитудный спектр записи, будет обеспечивать значительный рост энергии соответствующих спектральных компонент. Вполне вероятно, что всегда присутствующие в сейсмограммах случайные и когерентные шумы не имеют провала в спектре на этих частотах, поэтому применение оператора сигнатурной деконволюции приведет к неуправляемому росту амплитуды помех.

Хотя проявление таких помех зачастую носит локальный характер, однако даже из-за небольших некондиционных фрагментов приходится отбраковывать весь результат обработки, который оказался бы востребованным геофизиком. Поэтому желательно на этапе применения фильтра к данным производить адаптацию, контролируя уровень помех, которые не 
учитывались в процессе настройки фильтра, что повысит устойчивость процедуры. Настоящее исследование посвящено разработке такого алгоритма. В соответствии с изложенными выше принципами, алгоритм получил название устойчивая нестащионарная адаптивная сигнатурная деконволющия.

\section{Алгоритм}

Обозначим имеющуюся в распоряжении оценку формы импульса через $w(t)$, где $t$ - индекс дискретного времени. Желаемый импульс, к которому требуется преобразовать $w(t)$ в результате обработки, обозначим через $g(t)$. Искомый оператор такого преобразования - $f(t)$. Если в качестве критерия использовать традиционную для этой задачи меру в виде среднеквадратического отклонения фильтрованного сигнала $w(t)^{*} f(t)$ от желаемого (здесь звездочка обозначает свертку), то приходим к оптимизационной задаче

$$
f(t)=\underset{\tilde{f}(t)}{\arg \min } \sum_{t}(w(t) * \tilde{f}(t)-g(t))^{2},
$$

которая сводится к решению известной системы линейных уравнений с квадратной симметричной матрицей (Рапопорт, 1973). Для дальнейших рассуждений нам будет удобнее выписать спектральный аналог этого решения, который с учетом известных допущений записывается как:

$$
F(\omega)=G(\omega) / W(\omega)
$$

где $\omega$ - круговая частота, а через $W(\omega), \quad F(\omega)$ и $G(\omega)$ обозначены спектральные характеристики функций $w(t), f(t)$ и $g(t)$ соответственно. Для краткости условимся в дальнейшем называть $F(\omega)$ фильтром или оператором.

Вновь отметим, что оператор (1) (а также его частотный аналог (2)) записаны нами в рамках детерминированной постановки задачи. Тем не менее, его часто ошибочно называют винеровским фильтром, а поставленную выше задачу путают с задачей фильтрации случайных процессов.

Для реализации устойчивой нестационарной адаптивной сигнатурной деконволюции требуется производить дополнительную локальную по временной и латеральной координатам «подстройку» оператора (2) к зарегистрированному волновому полю. С этой целью используем скользящее окно прямоугольной формы, в котором будем получать оценку амплитудного спектра фрагмента волнового поля, которую для некоторого текущего положения окна обозначим как $|D(\omega)|$. Решение о наличии или отсутствии помехи, препятствующей получению достоверного результата сигнатурной деконволюции, будем принимать на основании сопоставления характеристик $|W(\omega)|$ и $|D(\omega)|$ на каждой частоте $\omega$ в пределах рабочего диапазона $\left(\omega_{1}, \omega_{2}\right)$. Очевидно, что эти характеристики могут отличаться по масштабу, поэтому алгоритм требует предварительного выравнивания коэффициента усиления амплитудных спектров $|W(\omega)|$ и $|D(\omega)|$. С этой целью выбирается диапазон частот $\left(\omega_{a}, \omega_{b}\right)$, в котором по результатам предварительного анализа волнового поля не ожидается появления помех. Вычисляется коэффициент $\gamma=\sum_{\omega_{a}}^{\omega_{b}}|W(\omega)| / \sum_{\omega_{a}}^{\omega_{b}}|D(\omega)|$, после чего производится нормировка $|\widetilde{D}(\omega)|=\gamma|D(\omega)|, \omega \in\left(\omega_{1}, \omega_{2}\right)$.

Рассчитанный ранее фильтр $f(t)$ следует скорректировать так, чтобы его применение не усиливало помеху. С этой целью используем пороговый алгоритм обнаружения помехи на каждой частоте в пределах рабочего диапазона. Выбрав величину порога $p$, производим сравнение: если $|\widetilde{D}(\omega)|<p|W(\omega)|$, то помехи нет. В противном случае принимается решение о 
наличии помехи. Во избежание ее возрастания в результате применения фильтрации амплитудный спектр оператора ограничивается сверху. Таким образом, амплитудный спектр преобразуется в соответствии со следующим правилом:

$$
|\widetilde{F}(\omega)|=\left\{\begin{array}{c}
|F(\omega)|, \text { если }|\widetilde{D}(\omega)|<p|W(\omega)| \\
p|F(\omega)| \frac{W(\omega) \mid}{|\widetilde{D}(\omega)|}, \text { если }|\widetilde{D}(\omega)| \geq p|W(\omega)|, \quad \omega \in\left(\omega_{1}, \omega_{2}\right),
\end{array}\right.
$$

при этом фазовый спектр фильтра $\widetilde{F}$ совпадает с фазовым спектром фильтра $F$. Полученная спектральная характеристика $\widetilde{F}$ соответствует оператору нестационарной адаптивной устойчивой фильтрации.

\section{Пример обработки}

Мы продемонстрируем возможности предложенного алгоритма, приведя пример обработки набора данных, полученных в результате морской сейсморазведки. При проведении наблюдений использовалась буксируемая коса, расположенная на глубине $\approx 15$ м. Источник находился на глубине $\approx 14 \mathrm{M}$. Таким образом, за счет влияния волн-спутников со стороны источника и приемника ожидается наличие провалов в спектральной характеристике сигнала на частотах соответственно $\approx 53$ Цц и $\approx 48$ ц. Для проведения сигнатурной деконволюции был рассчитан импульс (с этой целью использовалась специальная программа), и он показан на Рис. 1. Там же представлена оценка его амплитудного спектра. Очевидно, что импульс и спектр хорошо согласуются с ожидаемыми результатами. На этом же рисунке представлен фрагмент сейсмограммы в области первых вступлений, где сигнал уверенно выделяется, так как доминирует над помехами различной природы. Сравнив модельный и реальный импульсы, а также их амплитудные спектры, можно сделать вывод об их хорошем соответствии.

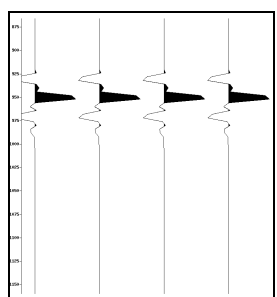

(a)

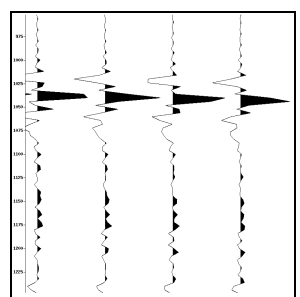

(б)

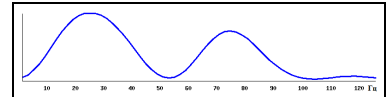

(в)

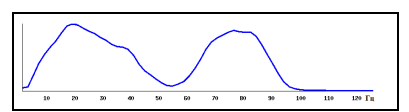

(г)

Рисунок 1 Сравнение модельного и реального сигналов. (a) импульс, полученный моделированием, (б) фрагмент исходной сейсмограммы, (в) амплитудный спектр модельного импульса, (г) амплитудный спектр показанного фрагмента волнового поля.

Вследствие наличия глубоко провала в амплитудном спектре импульса оператор сигнатурной деконволюции будет усиливать энергию этих гармоник. Присутствие помех на реальной сейсмограмме, не имеющих такого провала в спектре, приведет к значительному росту их энергии в окрестности указанных выше частот. Такое соображение подтверждается результатами обработки: на Рис. 2 сравниваются фрагменты сейсмограмм до и после сигнатурной деконволюции. На Рис. 3 представлены оценки амплитудных спектров, полученные по показанным фрагментам волновых полей. Очевидно, что использование устойчивой нестационарной адаптивной сигнатурной деконволюции позволяет преодолеть недостатки, характерные для этой процедуры.

Анализ волновой картины в окрестности первых вступлений по сейсмограмме после традиционной сигнатурной деконволюции с применением регуляризации (2\%) показывает, что оператор успешно преобразует сигнал, показанный на Рис. 1б, в нуль-фазовый импульс с равномерным в пределах рабочего диапазона частот амплитудным спектром. В то же время, как следует из Рис. 2б, этот же оператор искажает волновую картину в области больших удалений. Этот эффект свидетельствует о нестационарности характеристик исходного поля. Поэтому и оператор коррекции формы сигнала в условиях присутствия помех должен быть нестационарным и самонастраивающимся. В противном случае результат обработки будет 
забракован и, как часто бывает на практике, будет принято решение о недостаточной эффективности алгоритма сигнатурной деконволюции.

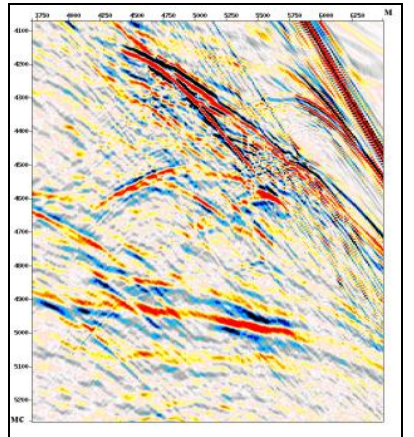

(a)

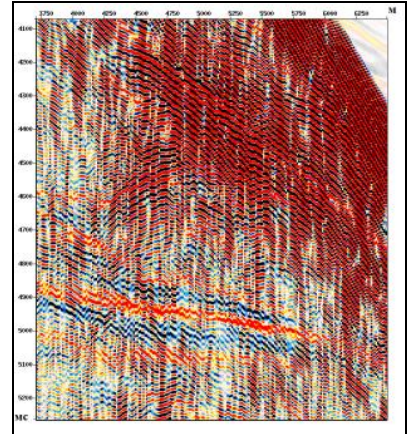

(б)

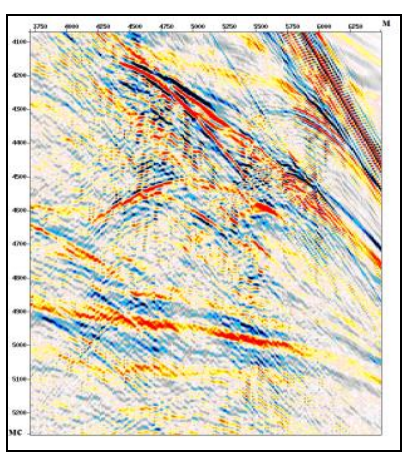

(в)

Рисунок 2 Фрагменты сейсмограмм. (а) исходное волновое поле. (б) результат традиционной сигнатурной деконволючии с регуляризащией $2 \%$. (в) результат устойчивой нестащионарной адаптивной сигнатурной деконволючии.

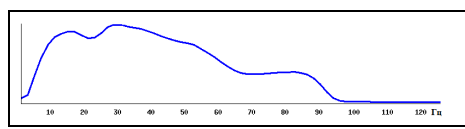

(a)

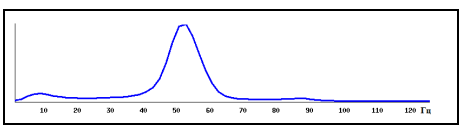

(б)

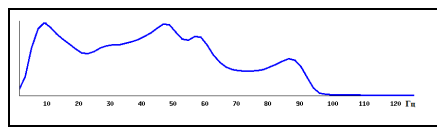

(B)

Рисунок 3 Оценки амплитудных спектров, полученные по показанным на Рис. 2 фрагментам волновых полей.

\section{Выводы}

Основной проблемой, с которой геофизик-обработчик сталкивается при применении сигнатурной деконволюции, является ее неустойчивость. Эта особенность алгоритма обусловлена тем, что настройка оператора производится по рассчитанной при помощи специальных программ или измеренной в процессе сейсморазведки форме импульса, а применяется этот оператор к сейсмограммам, которые могут быть осложнены помехами различной природы. С целью достижения устойчивости алгоритма нами предложен самонастраивающийся фильтр, обладающий возможностью локального (в пределах скользящего пространственно-временного окна) обнаружения помех на каждой частоте в пределах рабочего диапазона с последующей регулировкой усиления на этой частоте. Такой подход позволяет применять желаемый оператор сигнатурной деконволюции там, где это не приводит к росту амплитуды помех, и контролировать уровень шума в тех областях, где возможно его усиление. Эффективность алгоритма подтверждена результатом обработки набора данных, полученных в результате морской сейсморазведки.

Работа выполнена при финансовой поддержке Министерства образования и науки Российской Федерации (Программа СКИФ-НЕДРА, Государственный контракт № 14.964.11.0001 от 17 июня 2015 г.).

\section{Литература}

Боганик Г.Н., Гурвич И.И. [2006] Сейсморазведка. Тверь: АИС. 744 с.

Гогоненков Г.Н. [1987] Изучение детального строения осадочных толщ сейсморазведкой. М.: Недра. 222 с.

Рапопорт М.Б. [1973] Автоматическая обработка записей колебаний в сейсморазведке. М.: Недра. 184 с.

Хаттон Л., Уэрдингтон М., Мейкин Дж. [1989] Обработка сейсмических данных. Теория и практика. М.: Мир. 216 с. 


\section{EAGE}

Шехтман Г.А., Кузнецов В.М. [2005] Корреляция или деконволюция виброграмм - что лучше? // ВСП и трехмерные системы наблюдений в сейсморазведке: Гальперинские чтения-2005. М.: Geovers. C. 44-47.

\section{References}

Boganik G.N., Gurvich I.I. [2006] Seismoexploration. Tver: AIS. 744 p.

Gogonenkov G.N. [1987] Study of the detailed structure of sedimentary strata with seismic prospecting. Moscow: Nedra. 222 p.

Rapoport M.B. [1973] Automatic processing of oscillation records in seismic surveys. Moscow: Nedra. $184 \mathrm{p}$.

Hatton L., Worthington M.H., Makin J. [1989] Seismic data processing. Theory and practice. Moscow: Mir. 216 p.

Shekhtman G.A., Kuznetsov V.M. [2005] Correlation or deconvolution of vibrograms - what is better? // VSP and 3D seismic acquisitions systems: Galperinskie chtenija-2005. Moscow: Geovers. P. $44-47$. 